\title{
192.
}

\section{ON THE AREA OF THE CONIC SECTION REPRESENTED BY THE GENERAL TRILINEAR EQUATION OF THE SECOND DEGREE.}

[From the Quarterly Mathematical Journal, vol. II. (1858), pp. 248-253.]

[THE original title was "Direct Investigation of the Question discussed in the Foregoing Paper," viz. a Paper with the present title by N. M. Ferrers (now Dr Ferrers), pp. 247-248. The area $S$ of the conic section represented by the general equation $\left.\left(A, B, C, A^{\prime}, B^{\prime}, C^{\prime}\right\} x, y, z\right)^{2}=1$, where the coordinates are connected by the equation $x+y+z=1$, was by considerations founded on the form of the function found to be

$$
S=\frac{2 \pi\left(A A^{\prime 2}+B B^{\prime 2}+C C^{\prime 2}-A B C-2 A^{\prime} B^{\prime} C^{\prime}\right) \Delta}{\left\{A^{\prime 2}-B C+B^{\prime 2}-C A+C^{\prime 2}-A B+2\left(B^{\prime} C^{\prime}-A A^{\prime}\right)+2\left(C^{\prime} A^{\prime}-B B^{\prime}\right)+2\left(A^{\prime} B^{\prime}-C C^{\prime}\right)\right\}^{\frac{1}{2}}},
$$

where $\Delta$ is the area of the fundamental triangle: and it was remarked that a similar method might be applied to determine the area of the conic section when it is defined by the distances of its several tangents from three given points.]

The position of a point $P$ being determined as in the foregoing paper, let $\alpha, \beta, \gamma$ denote in like manner the coordinates of a point $O$, we have

$$
\alpha+\beta+\gamma=1,
$$

and consequently if $\xi, \eta, \zeta$ are the relative coordinates $x-\alpha, y-\beta, z-\gamma$, we have

$$
\xi+\eta+\zeta=0 .
$$


The expression for the distance of the two points $O, P$ is readily obtained in terms of the relative coordinates, viz. calling this distance $r$, we have

$$
r^{2}=L \xi^{2}+M \eta^{2}+N \xi^{2},
$$

where, if $l, m, n$ are the sides of the triangle $A B C$, we have

$$
\begin{aligned}
& L=\frac{1}{2}\left(m^{2}+n^{2}+l^{2}\right), \\
& M=\frac{1}{2}\left(n^{2}+l^{2}-m^{2}\right), \\
& N=\frac{1}{2}\left(l^{2}+m^{2}-n^{2}\right) ;
\end{aligned}
$$

and it is to be remarked that these values give

$$
M N+N L+L M=\frac{1}{4}\left(2 m^{2} n^{2}+2 n^{2} l^{2}+2 l^{2} m^{2}-l^{4}-m^{4}-n^{4}\right),=4 \Delta^{2},
$$

if $\Delta$ denote the area of the triangle $A B C$.

Consider now a conic

$$
\left(a, b, c, f, g, h \gamma(x, y, z)^{2},\right.
$$

and suppose as usual that $\mathfrak{A}, \mathfrak{B}, \mathfrak{E}, \mathfrak{F}, \mathfrak{E}, \mathfrak{S}$ are the inverse coefficients and that $K$ is the discriminant, suppose also for shortness

$$
P=(\mathfrak{A}, \mathfrak{B}, \mathfrak{( 5 ,} \mathfrak{F}, \mathfrak{F S}, \mathfrak{S} \gamma 1,1,1)^{2} .
$$

The coordinates of the centre being $\alpha, \beta, \gamma$, we have

$$
\begin{aligned}
& \alpha=\frac{1}{P}(\mathfrak{A}, \mathfrak{H}, \mathfrak{F} \gamma 1,1,1), \\
& \beta=\frac{1}{P}(\mathfrak{S}, \mathfrak{B}, \mathfrak{F} \gamma 1,1,1), \\
& \gamma=\frac{1}{P}(\mathfrak{S}, \mathfrak{F}, \mathfrak{F} \gamma 1,1,1),
\end{aligned}
$$

and writing as before $\xi, \eta, \zeta$ for $x-\alpha, y-\beta, z-\gamma$, so that $\xi, \eta, \zeta$ are the coordinates of a point $P$ of the conic, in relation to the centre, we have $x, y, z$ respectively equal to $\xi+\alpha, \eta+\beta, \zeta+\gamma$, and the equation of the conic gives

$$
(a, \ldots \gamma \xi+\alpha, \eta+\beta, \zeta+\gamma)^{2}=0,
$$

which may be written

$$
\begin{aligned}
& (a, \ldots \gamma \xi, \eta, \zeta)^{2} \\
+ & 2(a, \ldots \gamma \alpha, \beta, \gamma)(\xi, \eta, \zeta) \\
+ & \left(a, \ldots \gamma(\alpha, \beta, \gamma)^{2}=0 .\right.
\end{aligned}
$$


Now observing the equations

$$
\begin{aligned}
& \left(a, h, g \gamma(\alpha, \beta, \gamma)=\frac{K}{P},\right. \\
& \left(h, b, f \gamma(\alpha, \beta, \gamma)=\frac{K}{P},\right. \\
& \left(g, f, c \gamma(\alpha, \beta, \gamma)=\frac{K}{P},\right.
\end{aligned}
$$

we have

$$
\begin{aligned}
(a, \ldots \gamma(\alpha, \beta, \gamma)(\xi, \eta, \zeta) & =\frac{K}{P}(\xi+\eta+\zeta)=0, \\
\left(\alpha, \ldots \gamma(\alpha, \beta, \gamma)^{2}\right. & =\frac{K}{P}(\alpha+\beta+\gamma)=\frac{K}{P},
\end{aligned}
$$

and the equation of the conic gives therefore

$$
(a, \ldots \gamma \xi, \eta, \zeta)^{2}+\frac{K}{P}=0,
$$

and we have as before

$$
\xi+\eta+\zeta=0 .
$$

To find the axes we have only to make

$$
r^{2},=L \xi^{2}+M \eta^{2}+N \zeta^{2}
$$

a maximum or minimum, $\xi, \eta, \zeta$ varying subject to the preceding two conditions; this gives

$$
\begin{aligned}
& (a, h, g \gamma \xi, \eta, \zeta)+\lambda L \xi+\mu=0, \\
& (h, b, f \gamma \xi, \eta, \zeta)+\lambda M \eta+\mu=0, \\
& (g, f, c \gamma \xi, \eta, \zeta)+\lambda N \zeta+\mu=0,
\end{aligned}
$$

and multiplying by $\xi, \eta, \zeta$, adding and reducing, we have

$$
-\frac{K}{P}+\lambda r^{2}=0
$$

which gives

$$
\lambda=\frac{K}{P r^{2}} .
$$

Substituting this value, and joining to the resulting three equations the equation

$$
\xi+\eta+\zeta=0
$$

C. III. 
we may eliminate $\xi, \eta, \zeta, \mu$, and the result is

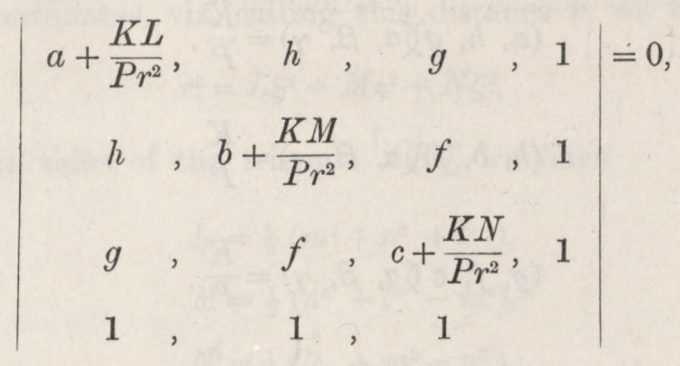

which may also be written

$$
\left(\mathfrak{A}^{\prime}, \mathfrak{B}^{\prime},\left(\mathfrak{C}^{\prime}, \mathfrak{F}^{\prime},\left(\mathfrak{S J}^{\prime}, \mathfrak{S}^{\prime} \mathfrak{\gamma} 1,1,1\right)^{2}=0,\right.\right.
$$

where $\left(\mathfrak{A}^{\prime}, \ldots\right)$ are what $(\mathfrak{A}, \ldots)$ become when $a, b, c$ are changed into

$$
a+\frac{K L}{P r^{2}}, \quad b+\frac{K M}{P r^{2}}, \quad c+\frac{K N}{P r^{2}}
$$

we in fact have

$$
\begin{aligned}
\mathfrak{A}^{\prime} & =\mathfrak{A}+\frac{K}{P r^{2}}(b N+c M)+\frac{K^{2}}{P^{2} r^{4}} M N, \\
: & \mathfrak{F}^{\prime}=\mathscr{F}-\frac{K}{P r^{2}} L f,
\end{aligned}
$$

and (observing the value of $P$ ) the result consequently is

$$
P+\frac{K}{P r^{2}}\{(b+c-2 f) L+(c+a-2 g) M+(a+b-2 h) N\}+\frac{K^{2}}{P^{2} r^{4}}(M N+N L+L M)=0,
$$

which may also be written

$$
P^{3} r^{4}+P K r^{2}\left\{\left(b+c-2 f^{\prime}\right) L+(c+a-2 g) M+(a+b-2 h) N\right\}+4 \Delta^{2} K^{2}=0 .
$$

Hence if $r_{1}, r_{2}$ are the two semiaxes, we have

$$
r_{1}^{2} r_{2}^{2}=\frac{4 \Delta^{2} K^{2}}{P^{3}}
$$

and the area is $\pi r_{1} r_{2}$ which is equal to

$$
\frac{2 \pi K \Delta}{\sqrt{ }\left(P^{3}\right)}
$$

which agrees with Mr Ferrers' result.

The formula $r^{2}=L \xi^{2}+M \eta^{2}+N \zeta^{2}$ which is assumed in the preceding investigation may be proved as follows: 
Writing $a, b, c$ (instead of $l, m, n$ ) for the sides of the fundamental triangle and $A, B, C$ for the angles, the equation in question is

$$
r^{2}=b c \cos A \xi^{2}+c a \cos B \eta^{2}+a b \cos C \zeta^{2} .
$$

Now writing $\alpha, \beta, \gamma$ for the inclinations of the line $r$ to the sides of the triangle, we have

$$
\begin{aligned}
& A=\beta-\gamma, \\
& B=\gamma-\alpha \\
& C=\pi+\alpha-\beta .
\end{aligned}
$$

Moreover taking for a moment $\lambda, \mu, \nu$ to denote the perpendiculars from the angles on the opposite sides, we have

$$
\begin{aligned}
& \lambda=c \sin B=b \sin C, \\
& \mu=a \sin C=c \sin A, \\
& \nu=b \sin A=a \sin B,
\end{aligned}
$$

and

$$
\xi=\frac{r \sin \alpha}{\lambda}, \quad \eta=\frac{r \sin \beta}{\mu}, \quad \zeta=\frac{r \sin \gamma}{\nu}
$$

the values of $\xi^{2}, \eta^{2}, \zeta^{2}$ consequently are

$$
\frac{r^{2} \sin ^{2} \alpha}{b c \sin B \sin C}, \frac{r^{2} \sin ^{2} \beta}{c a \sin C \sin A}, \quad \frac{r^{2} \sin ^{2} \gamma}{a b \sin A \sin B},
$$

and the equation to be proved becomes

$$
1=\frac{\cos A \sin ^{2} \alpha}{\sin B \sin C}+\frac{\cos B \sin ^{2} \beta}{\sin C \sin A}+\frac{\cos C \sin ^{2} \gamma}{\sin A \sin B}
$$

or, what is the same thing,

$\sin A \sin B \sin C=\sin A \cos A \sin ^{2} \alpha+\sin B \cos B \sin ^{2} \beta+\sin C \cos C \sin ^{2} \gamma$,

or again

$4 \sin A \sin B \sin C=\sin 2 A(1-\cos 2 \alpha)+\sin 2 B(1-\cos 2 \beta)+\sin 2 C(1-\cos 2 \gamma)$, or putting for $A, B, C$ their values in terms of $\alpha, \beta, \gamma$ this is

$$
\begin{aligned}
-4 \sin (\beta-\gamma) \sin (\gamma-\alpha) \sin (\alpha-\beta)= & \sin (2 \beta-2 \gamma)(1-\cos 2 \alpha) \\
& +\sin (2 \gamma-2 \alpha)(1-\cos 2 \beta) \\
& +\sin (2 \alpha-2 \beta)(1-\cos 2 \gamma)
\end{aligned}
$$


which is an identical equation; it is most readily proved by writing $x, y, z$ for $\tan \alpha, \tan \beta, \tan \gamma$; the equation thus becomes

$$
\begin{aligned}
& \frac{-4}{\left(1+x^{2}\right)\left(1+y^{2}\right)\left(1+z^{2}\right)}(y-z)(z-x)(x-y) \\
&=\Sigma \frac{1}{\left(1+y^{2}\right)\left(1+z^{2}\right)}\left\{2 y\left(1-z^{2}\right)-2 z\left(1-y^{2}\right)\right\} \frac{2 x^{2}}{1+x^{2}},
\end{aligned}
$$

or multiplying out

$$
-(y-z)(z-x)(x-y)=\Sigma(y-z)(1+y z) x^{2}=\Sigma x^{2}(y-z)+x y z \Sigma x(y-z),
$$

that is

$$
-(y-z)(z-x)(x-y)=\Sigma x^{2}(y-z) \quad=x^{2}(y-z)+y^{2}(z-x)+z^{2}(x-y),
$$

which is an identity.

[A different investigation of the formula $r^{2}=L \xi^{2}+M \eta^{2}+N \zeta^{2}$, by Dr Ferrers, was appended to the original Paper.] 